\section{Influence of muscle mass in the assessment of lower limb strength in COPD: validation of the prediction equation}

\begin{abstract}
Absence of established reference values limits application of quadriceps maximal voluntary contraction (QMVC) measurement. The impact of muscle mass inclusion in predictions is unclear. Prediction equations encompassing gender, age and size with (FFM+) and without (FFM-), derived in healthy adults ( $n=175)$, are presented and compared in two COPD cohorts recruited from primary care (COPD$P C, n=112$ ) and a complex care COPD clinic (COPD-CC, $n=189$ ). Explained variance was comparable between the prediction models (R2: FFM+: 0.59, FFM-: 0.60) as were per cent predictions in COPD-PC (88.8\%, 88.3\%). However, fat-free mass inclusion reduced the prevalence of weakness in COPD, particularly in COPD-CC where $11.9 \%$ fewer were deemed weak.
\end{abstract}

\section{INTRODUCTION}

Measurement of lower limb muscle strength is valuable in the clinical management of patients with COPD. Muscle weakness is common, independently relates to mortality and morbidity and is modifiable by exercise rehabilitation and potentially anabolic drug therapy. ${ }^{1-4}$ Assessment of lower limb strength can be easily and reproducibly performed in clinical settings through quadriceps maximal voluntary contraction (QMVC) measurement. Establishment of reference ranges in healthy adults is required to identify weakness to assist decisions regarding therapies and assess outcomes in both clinical and research settings. ${ }^{15}$ Prediction equations previously used to study strength in COPD populations have included fat-free mass (FFM) thereby incorporating a measure of muscle mass. The inclusion of FFM may underestimate the prevalence of muscle weakness, particularly in populations where muscle mass is frequently low, as in COPD. ${ }^{2}$ We aimed to examine the influence of muscle mass measurement on prediction equations for QMVC by determining the prevalence of weakness in two separate COPD cohorts using prediction equations with and without fat-free mass derived from healthy subjects.

\section{METHODS}

Prediction equations were derived using multiple linear regression from an existing cohort of healthy adults (HC). Age, gender, weight and height were entered in the first model. A whole-body measure of FFM was added for the second. The derived equations were used to calculate individual per cent predicted (\%pred) values of QMVC in two COPD cohorts: one recruited from primary care (COPD$\mathrm{PC})$ and the other from a complex care COPD outpatient clinic (COPD-CC). The lower limit of normal was used as a threshold for the presence of weakness. A further description of the participants, measurements and analysis is provided in the online supplementary file 1 .

\section{RESULTS}

One hundred and seventy-five HC participants were included and 301 patients with COPD $(n=112$, COPD-PC; $n=189$, COPD-CC). Baseline characteristics can be found in the table S1 in the online supplementary file 1 .

\section{Prediction models derived from healthy subjects}

Model without FFM (FFM-)

QMVC $=(-0.318 \times \mathrm{A})+(13.138$

$\times \mathrm{G})+(0.245 \times \mathrm{W})+(29.781 \times \mathrm{H})$ $-18.072$

QMVC (kg), A = age (years) $\mathrm{G}=$ gender: $(\mathrm{F}=0), \mathrm{W}=$ weight $(\mathrm{kg}), \mathrm{H}=$ height $(\mathrm{m})$

$$
\mathrm{R}=0.773, \mathrm{R}^{2}=0.598, \text { SEE: }
$$
$8.86, \mathrm{p} \leq 0.005$

\section{Model including FFM (FFM+)}

QMVC $=(-0.320 \times \mathrm{A})+(10.670 \times \mathrm{G})$

$+(0.566 \times$ FFM $)+20.952$

$\mathrm{FFM}=$ fat-free mass $(\mathrm{kg})$

$\mathrm{R}=0.770, \mathrm{R}^{2}=0.585, \mathrm{SEE}=8.90$, $\mathrm{p} \leq 0.005$

\section{Application of the prediction equations in patients with COPD}

The predicted values for QMVC using the FFM - and the FFM + model in the two COPD cohorts were calculated. Individual measured values were then compared with respective predictions as percentages to

Table 1 Quadriceps maximal voluntary contraction (QMVC) values expressed as per cent predicted (\%pred) values and number classed as weak using the FFM- and FFM + models for the COPD cohorts

\begin{tabular}{llc}
\hline & $\begin{array}{l}\text { Primary care COPD, } \\
\mathrm{n}=112\end{array}$ & $\begin{array}{l}\text { Complex care COPD, } \\
\mathrm{n}=189\end{array}$ \\
\hline FFM- model \%pred QMVC & $88.3(23.6)$ & $54.0(16.4)$ \\
\hline Number classed as weak (\%) & $17(15.2)$ & $101(53.4)$ \\
FFM+ model \%pred QMVC & $88.8(22.4)$ & $59.2(17.8)$ \\
Number classed as weak (\%) & $13(11.6)$ & $78(41.3)$ \\
\hline
\end{tabular}

Mean values and SD of measured QMVC presented as \%pred and the number in each cohort classed as weak using the FFM - and FFM+ models.

FFM+, fat-free mass model; FFM-, model without fat-free mass; $n$, number in each group.

yield the \%pred value for both models in all cohorts, presented in table 1 .

\section{QMVC weakness}

The number and proportion of each cohort classified as weak is presented in table 1 .

The FFM - model increased the percentage defined as weak $(3.6 \%$ increase in COPD-PC and $11.9 \%$ in COPD-CC) compared with the FFM+ model.

The distribution of the standardised residuals calculated using the FFM- and $\mathrm{FFM}+$ equations for the HC, primary care and complex care COPD cohorts in relation to the threshold of weakness are shown in figure 1.

\section{DISCUSSION}

We present two prediction equations for QMVC that estimate the presence of lower limb muscle weakness, one including and one without muscle mass (estimated using whole body measures of FFM). In healthy adults, inclusion of FFM did not affect explained variance of the prediction. However, when applied to COPD cohorts, there was a difference in the assessment of weakness between the two equations, which was amplified in those with more severe disease.

While percentage predicted values were similar in the primary care COPD (COPDPC) cohort using both equations there was a marginally greater number classed as weak with the FFM - model. A larger difference occurred between models in the complex care cohort (COPD-CC). Using the FFM+ model, \%pred values were higher and fewer were assigned as weak (table 1 and figure 1). This results from the partial adjustment for the lower muscle mass associated with more severe disease by the inclusion of FFM in the prediction equation. Where muscle mass is not abnormally low within the COPD$\mathrm{CC}$, the difference in the classification of weakness between the models is reduced (details of this subanalysis are supplied in 


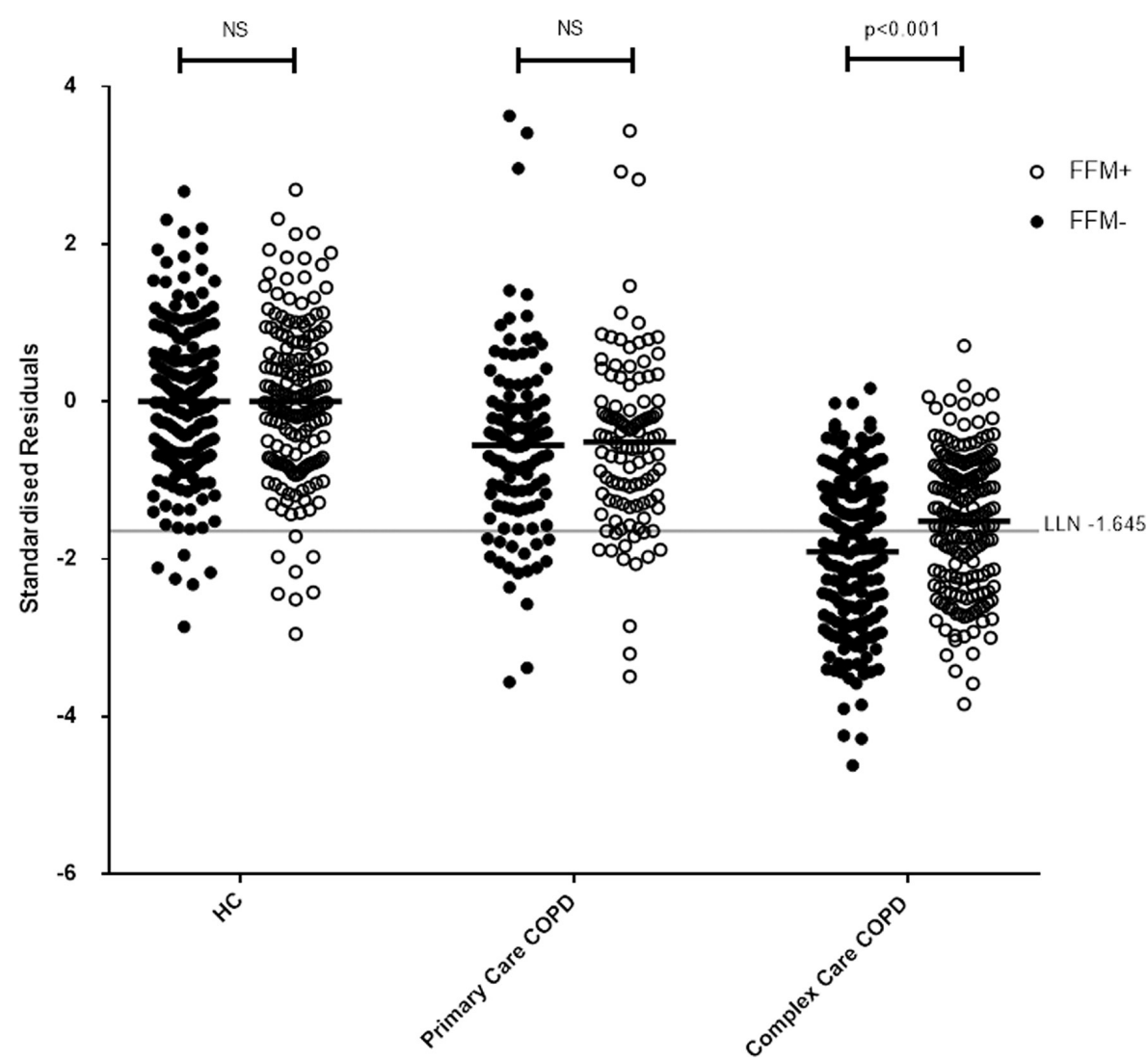

Figure 1 The distribution of the standardised residuals calculated using the FFM- and FFM+ equations for the $\mathrm{HC}$, primary care and complex care COPD cohorts. The lower limit of normal (LLN) threshold for weakness is shown as a grey horizontal line. Those below this line are classed as weak. Mean value for each group is shown as a solid black line. The significance of the difference between the standardised residuals for the models in each cohort is indicated above the respective columns. FFM-, model without fat-free mass; FFM+, fat-free mass included model; HC, healthy controls; NS, non-significant.

the online supplementary file 1 ).

The prevalence and magnitude of muscle weakness observed and the finding that this occurred in milder disease (managed in primary care) but was more pronounced in those with more severe COPD are consistent with other reports. ${ }^{246}$ Previous reference equations for QMVC have variably included muscle mass. ${ }^{24}$ A negligible difference in predictions with the inclusion of muscle mass in healthy subjects was reported in an examination of isokinetic muscle strength. ${ }^{7} \mathrm{~A}$ comparison of different reference equations for muscle strength in patients with COPD, one including FFM, demonstrated differences between them. ${ }^{8}$ We advance previous studies by directly comparing how model components influence predictions by using the same healthy cohort to derive equations and applying them to separate COPD cohorts of differing severities, from different healthcare sectors. We have identified statistical thresholds of 'normality' for muscle strength both in absolute terms and relative to an individual's muscle mass. The prediction does not encompass regional differences in muscle mass that might be important in some patients with COPD and clearly has relevance to the prediction of QMVC, a measure of regional muscle function. The impact of the identification of muscle weakness using this method on treatment stratification (eg, for local muscle reconditioning or whole body anabolic therapies) requires further investigation.

We acknowledge some limitations. Different methods were used to measure FFM in the two COPD cohorts, which could affect the predicted values but would have minimal effect on comparison of the prediction models. ${ }^{9}$ The functional and prognostic relevance of the identified lower limit of normal for muscle strength requires confirmation through linkage with outcomes such as functional status and mortality.

Proximal lower limb muscle dysfunction has significant implications for mortality, morbidity and healthcare utilisation in COPD. ${ }^{4}$ Measurement is important in clinical assessment with the potential to aid targeting of therapeutic interventions such as strength training, nutritional support and anabolic drug therapy, availability of accessible reference values for interpretation will assist implementation. ${ }^{10}$

Ruth Trethewey, ${ }^{1}$ Dale Esliger, ${ }^{1,2}$

Emily Petherick ${ }^{1}$ Rachael Evans, ${ }^{1,3,4}$

Neil Greening, ${ }^{3,5}$ Benjamin James, ${ }^{5}$

Andrew Kingsnorth, ${ }^{1}$ Mike Morgan, ${ }^{3}$

Mark Orme, ${ }^{1}$ Lauren Sherar, ${ }^{1}$ Sally Singh, ${ }^{1,4}$

Nicole Toms, ${ }^{3,5}$ Michael Steiner ${ }^{1,4,5}$

${ }^{1}$ School of Sport, Exercise and Health Sciences, National Centre for Sport and Exercise Medicine, Loughborough University, Loughborough, UK

${ }^{2}$ NIHR Leicester Biomedical Research Centre - Lifestyle, Leicester, UK

${ }^{3}$ Respiratory Medicine, Glenfield Hospital, Leicester, UK ${ }^{4}$ Centre for Exercise and Rehabilitation Services, University Hospitals of Leicester NHS Trust, Leicester, UK ${ }^{5} \mathrm{NIHR}$ Leicester Biomedical Research Centre Respiratory, Leicester, UK

Correspondence to Ruth Trethewey, National Centre for Sport and Exercise Medicine, School of Sport Exercise and Health Sciences, Loughborough LE11 3TU, UK; R.Pearson3@|boro.ac.uk

Acknowledgements The research was supported by National Health Service (NHS) England. The authors acknowledge support from the National Institute for Health Research (NIHR) Leicester Biomedical Research Centre, which is a partnership between University Hospitals of Leicester NHS Trust, Loughborough University and the University of Leicester; the NIHR Collaboration for Leadership in Applied Health Research and Care East Midlands (NIHR CLAHRC EM) and The Primary Care Research Network. The Physical Activity and Respiratory Health study was funded by the Department of Health. The views expressed are those of the authors and not necessarily those of NHS England, the NIHR, Loughborough University or the Department of Health.

Contributors RET and MCS developed the concept of the study and wrote the manuscript. Others contributed to the final version. RET, ESP, DWE, NJG and MCS were involved in the study design and data analysis. BDJ made additional contributions to the data analysis. DWE, RAE, NJG, AK, MWO, LBS, SJS, MCS and NT were also involved in the data acquisition and all authors have provided approval of the final version to be published.

Competing interests None declared.

Ethics approval The cohorts studied had the relevent NHS REC approvals: 13-EM-0389, 13/ EM/0287.

Provenance and peer review Not commissioned; externally peer reviewed.

(C) Article author(s) (or their employer(s) unless otherwise stated in the text of the article) 2018. All rights reserved. No commercial use is permitted unless otherwise expressly granted.

- Additional material is published online only. To view please visit the journal online (http://dx.doi.org/ 10.1136/thoraxjnl-2016-209870).

\section{Check for updates}

To cite Trethewey R, Esliger D, Petherick E, et al. Thorax 2018;73:587-589. 
Received 15 December 2016

Revised 24 July 2017

Accepted 14 August 2017

Published Online First 2 September 2017

Thorax 2018:73:587-589

doi:10.1136/thoraxjnl-2016-209870

\section{REFERENCES}

1 Maltais F, Decramer M, Casaburi R, et al. An official American Thoracic Society/European Respiratory Society statement: update on limb muscle dysfunction in chronic obstructive pulmonary disease. Am J Respir Crit Care Med 2014:189:e15-e62.

2 Seymour JM, Spruit MA, Hopkinson NS, et al. The prevalence of quadriceps weakness in COPD and the relationship with disease severity. Eur Respir J 2010;36:81-8.

3 Swallow EB, Reyes D, Hopkinson NS, et al. Quadriceps strength predicts mortality in patients with moderate to severe chronic obstructive pulmonary disease. Thorax 2007:62:115-20.

4 Decramer M, Gosselink R, Troosters T, et al. Muscle weakness is related to utilization of health care resources in COPD patients. Eur Respir J 1997:10:417-23.

5 Greening NJ, Williams JE, Hussain SF, et al. An early rehabilitation intervention to enhance recovery during hospital admission for an exacerbation of chronic respiratory disease: randomised controlled trial. BMJ 2014;349:g4315.

6 Bernard S, LeBlanc P, Whittom F, et al. Peripheral muscle weakness in patients with chronic obstructive pulmonary disease. Am J Respir Crit Care Med 1998:158:629-34.

7 Neder JA, Nery LE, Shinzato GT, et al. Reference values for concentric knee isokinetic strength and power in nonathletic men and women from 20 to 80 years old. J Orthop Sports Phys Ther 1999:29:116-26.

8 Nellessen AG, Donária L, Hernandes NA, et al. Analysis of three different equations for predicting quadriceps femoris muscle strength in patients with COPD. J Bras Pneumol 2015:41:305-12.

9 Steiner MC, Barton RL, Singh SJ, et al. Bedside methods versus dual energy X-ray absorptiometry for body composition measurement in COPD. Eur Respir J 2002;19:626-31.

10 Cielen N, Maes K, Gayan-Ramirez G. Musculoskeletal disorders in chronic obstructive pulmonary disease. Biomed Res Int 2014:2014:1-17. 